TRANSACTIONS OF THE

AMERICAN MATHEMATICAL SOCIETY

Volume 353, Number 1, Pages 191-208

S 0002-9947(00)02465-X

Article electronically published on June 8, 2000

\title{
BLOW UP AND INSTABILITY OF SOLITARY-WAVE SOLUTIONS TO A GENERALIZED KADOMTSEV-PETVIASHVILI EQUATION
}

\author{
YUE LIU
}

\begin{abstract}
In this paper we consider a generalized Kadomtsev-Petviashvili equation in the form

$$
\left(u_{t}+u_{x x x}+u^{p} u_{x}\right)_{x}=u_{y y} \quad(x, y) \in R^{2}, t \geq 0 .
$$

It is shown that the solutions blow up in finite time for the supercritical power of nonlinearity $p \geq 4 / 3$ with $p$ the ratio of an even to an odd integer. Moreover, it is shown that the solitary waves are strongly unstable if $2<p<4$; that is, the solutions blow up in finite time provided they start near an unstable solitary wave.
\end{abstract}

\section{INTRODUCTION}

We are concerned in this paper with a generalized Kadomtsev-Petviashvili (KP) equation; that is,

$$
\left\{\begin{array}{l}
u_{t}+u_{x x x}+u^{p} u_{x}=v_{y} \\
v_{x}=u_{y}
\end{array} \quad(x, y) \in \mathbb{R}^{2}, t \geq 0 .\right.
$$

When $p=1$, the equation (1.1) is known as the KP-I equation. It is a model for the propagation of weakly nonlinear dispersive long waves on the surface of a fluid, when the wave motion is essentially one-directional with weak transverse effects along the $y$-axis $\mathrm{KaPe}, \mathrm{PeYa}$. The KP-I equation has been analyzed in great detail. It can be solved by the Inverse Scattering Transformation (IST) AbSe, FoSu, Zh. When $p=2$, the equation (1.1) is also a model to describe the evolution of sound waves in antiferromagnetics $\mathrm{TuFa}$. To some extent, the $\mathrm{KP}$ equation can be viewed as a two-dimensional analog of the generalized Korteweg-de Vries (GKdV) equation.

Many rigorous results have recently appeared concerning the Cauchy problem for the KP equation Bou, FoSu, IsMeSt, Sa1 Sa2, Uk, Zh, To. A general local existence theory has been established by Ukai [Uk] and developed by Saut [Sa1] in the classical Sobolev space $H^{s}\left(\mathbb{R}^{2}\right)$ for $s \geq 3$. As we know, one of the more challenging issues concerning the Cauchy problem for nonlinear dispersive equations is the possible blow-up of solutions in finite time. For instance, consider the GKdV equation

$$
u_{t}+u_{x x x}+u^{p} u_{x}=0
$$

Received by the editors April 6, 1998 and, in revised form, September 2, 1998.

2000 Mathematics Subject Classification. Primary 35Q53, 35B60, 76B25.

(C)2000 American Mathematical Society 
Though numerical simulations BoDoKa] do show that blow-up can occur for $p \geq 4$, a rigorous proof of the blow-up for $p \geq 4$ is still missing. Compared with GKdV, the KP equation does have a blow-up solution if the energy of the initial data is negative and the power of nonlinearity $p \geq 4$. This has been proved by using a virial identity TuFa, Sa1]. On the other hand, by using the anisotropic Sobolev embedding

$$
\int_{\mathbb{R}^{2}}|u|^{p+2} d x d y \leq c\|u\|_{L^{2}}^{\frac{4-p}{2}}\left\|\partial_{x} u\right\|_{L^{2}}^{p}\|v\|_{L^{2}}^{\frac{p}{2}}
$$

which is valid only for $0 \leq p \leq 4$, we can deduce that $\int\left(\left(u_{x}\right)^{2}+v^{2}\right) d x d y$ is dominated by the conserved momentum $V(u)=\frac{1}{2} \int_{\mathbb{R}^{2}} u^{2} d x d y$ and the Hamiltonian

$$
E(u)=\int_{\mathbb{R}^{2}}\left(\frac{1}{2} u_{x}^{2}+\frac{1}{2} v^{2}-\frac{1}{(p+1)(p+2)} u^{p+2}\right) d x d y
$$

if and only if $p<\frac{4}{3}$. It is also shown by some numerical simulations Wi, WaAbSe that there are blow-up solutions for the KP equation (1.1) in finite time for $p \geq \frac{4}{3}$. Therefore, it is natural to conjecture that the solutions of the KP equation (1.1) should blow up in finite time for $p \geq \frac{4}{3}$. The purpose of this paper is to investigate questions about blow-up solutions and instability of solutions for (1.1). We are able to show that the solution of (1.1) blows up in finite time for $p \geq \frac{4}{3}$ (Theorems 4.1, 4.2 ). It turns out that the power of $p=\frac{4}{3}$ is the critical power of the nonlinearity for the existence of solutions. The critical power $p=\frac{4}{3}$ is also observed in the study of stability and instability of solitary waves. As we know, in the case of the GKdV equation, the power $p=4$ is the critical value for the stability and instability of the solitary waves $\varphi_{c}(x-c t)$ We, BoSoSt SoSt. As in the case of the GKdV equation, it is found that the critical power $p=\frac{4}{3}$ is also linked to the stability and instability of the solitary-wave solutions $\varphi_{c}(x-c t, y)$ for (1.1) (see Definition 2.5). Indeed, the solitary-wave solutions vanishing in the infinity exist if and only if $1 \leq p<4$ WaAbSe, Sa1, Sa2. It is shown that the set of the solitary waves $\varphi_{c}$ with $c>0$ is nonlinearly stable for $1 \leq p<\frac{4}{3}$ [LiuWa, BoSa3] and it is nonlinearly unstable for $\frac{4}{3}<p<4$ [WaAbSe, BoSa3]. Therefore, it is reasonable to conjecture that the solution of the KP equation (1.1) blows up in finite time for $p \geq \frac{4}{3}$ provided it begins near the solitary-wave solution $\varphi_{c}$ (i.e., strong instability). In this paper, we also give a satisfactory result to solve this strong instability problem (Theorem 5.1). More interestingly, it is proved in Theorem 5.1 that for the power $2<p<4$, solutions with initial data close to unstable solitary waves are actually bounded for all time in energy space. However, they do blow up in finite time in some sense $\left(\left\|\partial_{y} u(\cdot, t)\right\|_{L^{2}} \rightarrow \infty\right.$ in finite time $)$, due to the weak transverse dispersion along the $y$-axis.

In general, solutions of (1.1) satisfy the following conserved functionals.

$$
\begin{gathered}
E(u)=\int_{\mathbb{R}^{2}}\left(\frac{1}{2} u_{x}^{2}+\frac{1}{2} v^{2}-\frac{1}{(p+1)(p+2)} u^{p+2}\right) d x d y \quad \text { (Energy) } \\
V(u)=\frac{1}{2} \int_{\mathbb{R}^{2}} u^{2} d x d y \quad \text { (Momentum) } \\
\Phi_{1}(u)=\int_{\mathbb{R}^{2}} u d x d y \quad \text { and } \quad \Phi_{2}(u)=\int_{\mathbb{R}^{2}} v d x d y
\end{gathered}
$$


Also, in the case of $p=1$,

$$
\Phi_{3}(u)=\int_{\mathbb{R}^{2}}\left(\frac{3}{2} u_{x x}^{2}+5 u_{y}^{2}+\frac{5}{6}\left(\partial_{x}^{-1} v_{y}\right)^{2}-\frac{5}{2} u u_{x}^{2}-\frac{5}{9} u v^{2}\right) d x d y
$$

is conserved. In fact, for $p=1$, there are infinitely many conservation laws.

In this paper, we shall employ the following notations. $|\cdot|_{p}$ and $\|\cdot\|_{s}$ will denote the norms in $L^{p}\left(\mathbb{R}^{2}\right)$ and Sobolev space $H^{s}\left(\mathbb{R}^{2}\right)$ respectively. And we will borrow the notations in [Sa1, Sa2]. Let $Y$ be the closure of $\partial_{x}\left(C_{0}^{\infty}\left(\mathbb{R}^{2}\right)\right)$ with the norm

$$
\|u\|_{Y}=\left\|\partial_{x} \psi\right\|_{Y}=\left(\|\nabla \psi\|_{L^{2}}^{2}+\left\|\partial_{x}^{2} \psi\right\|_{L^{2}}^{2}\right)^{\frac{1}{2}}
$$

for $u \in Y$ and $u=\partial_{x} \psi$, where $\psi \in L_{l o c}^{q}\left(\mathbb{R}^{2}\right), \forall 2 \leq q<\infty$. We also have $v=\partial_{y} \psi \in$ $L^{2}$ by a choice of $\psi \in L_{l o c}^{q}$. Let

$$
\begin{gathered}
X=\left\{u \in H^{1}\left(\mathbb{R}^{2}\right) ; \partial_{x}^{2} u \in L^{2}\right\}, \\
X_{s}=\left\{u \in H^{1}\left(\mathbb{R}^{2}\right) ;\left(\xi_{1}^{-1} \hat{u}\right)^{\vee} \in H^{s}\left(\mathbb{R}^{2}\right)\right\}
\end{gathered}
$$

with the norm $\|u\|_{X_{s}}=\|u\|_{s}+\left\|\left(\xi_{1}^{-1} \hat{u}\right)^{\vee}\right\|_{s}$ and

$$
\dot{H}^{-k}=\left\{u \in S^{\prime}\left(\mathbb{R}^{2}\right), \xi_{1}^{-k} \hat{u} \in L^{2}\left(\mathbb{R}^{2}\right)\right\}
$$

equipped with the norm $\|u\|_{-k, x}=\left|\xi_{1}^{-k} \hat{u}\right|_{2}$, where " $\wedge$ " is the Fourier transform, " $\vee$ " is the Fourier inverse transform and $\xi=\left(\xi_{1}, \xi_{2}\right)$ is the dual variable of $(x, y)$.

Throughout this paper, we only consider the case when $p=\frac{n_{1}}{n_{2}}$ where $n_{1}$ is any even integer and $n_{2}$ any odd integer so that $\int_{\mathbb{R}^{2}} u^{p+2} d x d y=|u|_{p+2}^{p+2}$.

The main aim of the paper is to investigate properties of blow-up and instability of the solitary-wave solutions $\varphi_{c}$ for the critical value $p \geq \frac{4}{3}$. The main theorems in the paper are Theorem 4.1 and Theorem 5.1. The key ingredients used here are to construct some virial identities, and study the minimization problems which are related to the solitary waves.

The plan of this paper is as follows. In Section 2, we study the properties of the solitary-wave solutions $\varphi_{c}$. In particular, we consider the associated minimization problem and employ a refined Fatou lemma due to Lieb and Brézis BrLi1 to obtain the suitable minimizer, which is also known as "ground state." In Section 3, some invariant sets for the flow of the KP equation are constructed. With the help of the invariant sets, we are able to establish the blow-up results in the super critical case $p>\frac{4}{3}$, and a detailed proof is given in Section 4 . In the last section, a strong instability of solitary-wave solution is investigated. It is proved that for $2<p<4$ the solution of (1.1) blows up in finite time provided it starts close to a solitary-wave solution.

\section{Solitary-WAVE SOLUTiOns}

In this section, we study some important properties of the solitary-wave solution $\varphi_{c}$ to the KP equation (1.1). By a solitary-wave solution of (1.1), we mean a traveling-wave solution of (1.1) in the form $u(t, x, y)=\varphi_{c}(x-c t, y)$ with $u \rightarrow 0$ as $x^{2}+y^{2} \rightarrow \infty$. And $\varphi_{c}$ is a ground state of the equation

$$
\left\{\begin{array}{l}
-c \varphi_{c}+\partial_{x}^{3} \varphi_{c}-\partial_{y} \psi_{c}+\frac{1}{p+1} \varphi_{c}^{p+1}=0, \\
\partial_{x} \psi_{c}=\partial_{y} \varphi_{c} \quad \varphi_{c} \in Y, \varphi_{c} \neq 0 .
\end{array}\right.
$$

Let $L_{c}(u)=E(u)+c V(u)$. We define the ground states of (2.1) as follows. 
Definition 2.1. Let $\Gamma_{c}$ be the set of the solutions of (2.1); namely,

$$
\Gamma_{c}=\left\{\phi \in Y \mid L_{c}^{\prime}(\phi)=0, \phi \neq 0\right\},
$$

and let $G_{c}$ be the set of the ground states of (2.1); that is

$$
G_{c}=\left\{\varphi \in \Gamma_{c} \mid L_{c}(\varphi) \leq L_{c}(\phi), \forall \phi \in \Gamma_{c}\right\} .
$$

Remark 2.2. The existence of ground states for (2.1) was proved in WaAbSe, [BoSa]. It is shown that $G_{c}$ is not empty for any $c>0$ and $1 \leq p<4$. But the uniqueness of the ground state is still unknown.

Remark 2.3. For $p=1$, there exists an explicit traveling-wave solution of the KP equation (1.1), which is called lump soliton $\phi_{c}(x-c t, y)$ with $c>0$ AbSe, where

$$
\phi_{c}(x, y)=\frac{8 c\left(1-\frac{c}{3} x^{2}+\frac{c^{2}}{3} y^{2}\right)}{\left(1+\frac{c}{3} x^{2}+\frac{c^{2}}{3} y^{2}\right)^{2}} .
$$

However, to our knowledge, it is still an open problem whether or not the lump is a ground state.

There are many results on local existence to the KP equation (1.1). For our purpose, we state here the local existence result by Saut [Sa1].

Lemma 2.4. Let $u_{0} \in X_{s}, s \geq 3$, and $\partial_{y y}^{2} u_{0} \in \dot{H}_{x}^{-2}$. Then there exists $T>0$ such that the equation (1.1) has a unique solution $u(t)$ with $u(0)=u_{0}$ satisfying

$$
u \in C\left([0, T) ; H^{s}\left(\mathbb{R}^{2}\right)\right) \cap C^{1}\left([0, T) ; H^{s-3}\left(\mathbb{R}^{2}\right)\right)
$$

and

$$
v \in C\left([0, T) ; H^{s-1}\left(\mathbb{R}^{2}\right)\right) .
$$

Moreover, the energy $E(u)$ and the momentum $V(u)$ are well defined and independent of time $t$.

Next we state the definition of stability of the solitary waves in the following.

Definition 2.5. We say that a set $S \subset X$ is $X$-stable, if for any $\epsilon>0$, there exists $\delta>0$ with $\inf _{w \in S}\left\|u_{0}-w\right\|_{X}<\delta$ for any $u_{0} \in X \cap X_{s}$ and $\partial_{y y}^{2} u_{0} \in \dot{H}_{x}^{-2}, s \geq 3$; the solution $u(t)$ of (1.1) with $u(0)=u_{0}$ can be extended to a global solution in $C\left([0, \infty) ; X \cap X_{3}\right)$ and satisfies

$$
\sup _{0 \leq t<\infty} \inf _{w \in S}\|u(t)-w\|_{X}<\epsilon .
$$

Otherwise, $S$ is called $X$-unstable. Also we say that the solitary wave $\varphi_{c}$ is unstable if $O_{c}=\left\{\tau_{s, r} u \quad \mid s, r \in \mathbb{R}^{2}, \tau_{s, r} u(x, y)=u(x+s, y+r)\right\}$ is $O_{c^{-} \text {unstable. Further- }}$ more, we say that $\varphi_{c}$ is strongly unstable if for any $\epsilon>0$, there exists $u_{0} \in Y$ such that the solution $u(t)$ of (1.1) with $u(0)=u_{0}$ blows up in finite time.

Remark 2.6. The set of ground states $G_{c}$ with $c>0$ for (1.1) is $Y$-stable provided $1 \leq p<\frac{4}{3}$ is proved in [LiuWa, BoSa3] and $G_{c}$ is $Y$-unstable is also proved in WaAbSe, BoSa3] if $\frac{4}{3}<p<4$.

To show blow up and strong instability of solutions of (1.1), we need a series of lemmas.

The first lemma is called refined Fatou lemma due to Brézis and Lieb BrLi1] which is crucial to solve a constrained minimization problem (Lemma 2.11). 
Lemma 2.7. Let $\left\{f_{j}\right\}$ be a bounded sequence in $L^{r}\left(\mathbb{R}^{2}\right)$ for $0<r<\infty$. If $f_{j} \rightarrow f$ a.e. in $\mathbb{R}^{2}$, then

$$
\left|f_{j}\right|_{r}^{r}-\left|f_{j}-f\right|_{r}^{r}-|f|_{r}^{r} \longrightarrow 0 .
$$

When $r=2$, the assumption that $f_{j} \rightarrow f$ a.e. is not necessary.

The following lemma is given in [BoSa1], Lemma 3.3.

Lemma 2.8. Let $u_{n}$ be a bounded sequence in $Y$, and let $R>0$. Then there is a subsequence $u_{n_{k}}$ which converges strongly to some $u$ in $L^{2}\left(B_{R}\right)$, where $B_{R}$ is a ball with radius $R$ in $\mathbb{R}^{2}$.

Lemma 2.9. Let $u \in Y$ such that $\|u\|_{Y} \leq C$ and $\mu(|u|>\epsilon) \geq \delta>0$. Then there exists a shift $\tau_{s, r} u(x, y)=u(x+s, y+r)$, such that for some constant $\delta_{0}=$ $\delta_{0}(c, \delta, \epsilon)>0$,

$$
\mu\left(B \cap\left(\left|\tau_{s, r} u\right|>\frac{\epsilon}{2}\right)\right)>\delta_{0}
$$

where $B$ is the unit ball in $\mathbb{R}^{2}$.

Proof. See Lemma 4 in LiuWa with the space $Y$.

Lemma 2.10. Suppose $1 \leq p<4$ and $c>0$. Let $\varphi_{c}$ be a ground state. Then

a) $0=K\left(\varphi_{c}\right)=\inf \left\{K(u) \mid u \in Y, \int_{\mathbb{R}^{2}}\left(\partial_{x} u\right)^{2}=\int_{\mathbb{R}^{2}}\left(\partial_{x} \varphi_{c}\right)^{2}\right\}$;

b) $I_{c}\left(\varphi_{c}\right)=\inf \left\{I(u) \mid u \in Y, \int_{\mathbb{R}^{2}}(u)^{p+2}=\int_{\mathbb{R}^{2}}\left(\varphi_{c}\right)^{p+2}\right\}$,

where

$$
\begin{aligned}
K(u) & =\frac{1}{2} \int_{\mathbb{R}^{2}}\left(c u^{2}+v^{2}\right)-\frac{1}{(p+1)(p+2)} \int_{\mathbb{R}^{2}} u^{p+2}, \\
K\left(\varphi_{c}\right)=0 \text { and } I_{c}(u) & =\int_{\mathbb{R}^{2}}\left(c u^{2}+v^{2}+\left(\partial_{x} u\right)^{2}\right) d x d y .
\end{aligned}
$$

Proof. See Lemma 2.1 in [BoSa1] or Theorem 1 in LiuWa].

Lemma 2.11. Let $\sigma=\inf \left\{L_{c}(u) \mid u \in M\right\}$, where

$$
M=\{u \in Y \mid u \neq 0, Q(u)=0\}
$$

and

$$
Q(u)=\int_{\mathbb{R}^{2}}\left(v^{2}+\left(\partial_{x} u\right)^{2}-\frac{3 p}{2(p+1)(p+2)} u^{p+2}\right) d x d y .
$$

Assume $\frac{4}{3}<p<4$. Then $\varphi_{c}$ is a ground state for (2.1) if and only if $\varphi_{c} \in M$ and $L_{c}\left(\varphi_{c}\right)=\sigma$.

Proof. Let $\varphi_{c}$ be a ground state and define $\phi_{c}^{\lambda}(x, y)=\lambda^{\frac{3}{2}} \varphi_{c}\left(\lambda x, \lambda^{2} y\right)$. It is easy to see that

$$
Q\left(\varphi_{c}\right)=\left.\frac{d}{d \lambda} L_{c}\left(\phi_{c}^{\lambda}\right)\right|_{\lambda=1}=\left\langle L_{c}^{\prime}\left(\varphi_{c}\right),\left.\frac{d \phi_{c}^{\lambda}}{d \lambda}\right|_{\lambda=1}\right\rangle=0 .
$$

This implies that $\varphi_{c} \in M$ for any $\varphi_{c} \in G_{c}$. Now we define

$$
L_{c}^{1}(u)=L_{c}(u)-\frac{2}{3 p} Q(u)=\frac{c}{2} \int_{\mathbb{R}^{2}} u^{2}+\frac{3 p-4}{6 p} \int_{\mathbb{R}^{2}}\left(v^{2}+u_{x}^{2}\right) .
$$


We claim $\sigma=m$, where

$$
m=\inf \left\{L_{c}^{1}(u) \mid u \in Y, u \neq 0, Q(u) \leq 0\right\} .
$$

In fact, suppose $Q(u)<0$. Since

$$
Q(\lambda u)=\lambda^{2} \int_{\mathbb{R}^{2}}\left(v^{2}+u_{x}^{2}\right)-\frac{3 p}{2(p+1)(p+2)} \lambda^{p+2} \int_{\mathbb{R}^{2}} u^{p+2}>0
$$

for some sufficiently small $\lambda>0$, there exists $\lambda_{0} \in(0,1)$ such that $Q\left(\lambda_{0} u\right)=0$. Hence we have

$$
\begin{aligned}
\sigma \leq L_{c}\left(\lambda_{0} u\right) & =\lambda_{0}^{2}\left(\frac{c}{2} \int_{\mathbb{R}^{2}} u^{2}+\frac{3 p-4}{6 p} \int_{\mathbb{R}^{2}}\left(v^{2}+u_{x}^{2}\right)\right) \\
& <\frac{c}{2} \int_{\mathbb{R}^{2}} u^{2}+\frac{3 p-4}{6 p} \int_{\mathbb{R}^{2}}\left(v^{2}+u_{x}^{2}\right)=L_{c}^{1}(u) .
\end{aligned}
$$

Consequently, $m=\sigma$. To show that $\sigma=L_{c}\left(\varphi_{c}\right)$ for some $\varphi_{c} \in G_{c}$, it suffices to show that $m=L^{1}\left(\varphi_{c}\right)$ for some $\varphi_{c} \in G_{c}$. In fact, we have $L_{c}^{1}(u)>0$ for $\frac{4}{3}<p<4$. Hence, there exists a minimizing sequence $\left\{u_{j}\right\}$ of (2.7) satisfying that $\left\{u_{j}\right\}$ is bounded in $Y, L_{c}^{1}\left(u_{j}\right) \rightarrow m$ and $Q\left(u_{j}\right) \leq 0$. By the anisotropic Sobolev embedding BeIINi p. 323],

$$
|u|_{p+2} \leq c\|u\|_{Y} \quad \text { for } 0 \leq p \leq 4 .
$$

It turns out that $\left\{u_{j}\right\}$ is bounded in $L^{p+2}$ for $0 \leq p<4$. We then have some subsequences, still denoted by $\left\{u_{j}\right\}$ and $u_{0} \in Y \cap L^{p+2}$ such that $u_{j}$ weakly converges to $u_{0}$ in $Y$ and in $L^{p+2}$ for $0<p<4$. It follows from Lemma 2.8 that $u_{j} \rightarrow u_{0}$ a.e. in $\mathbb{R}^{2}$. Now we are able to show $L_{c}^{1}\left(u_{0}\right)=m$ and $Q\left(u_{0}\right)=0$. Toward this end, we split the proof into the following five steps.

Step 1. $\inf _{j}\left|u_{j}\right|_{p+2}^{p+2}>0$.

Proof of Step 1. Suppose there exists a subsequence of $\left\{u_{j}\right\}$ such that $\left|u_{j}\right|_{p+2}^{p+2} \rightarrow 0$. Then from $Q\left(u_{j}\right) \leq 0$ we obtain

$$
\left|v_{j}\right|_{2}^{2}+\left|\partial_{x} u_{j}\right|_{2}^{2} \rightarrow 0
$$

On the other hand, by the facts that $Q\left(u_{j}\right) \leq 0$ and the anisotropic Sobolev embedding [To, we have

$$
|u|_{p+2}^{p+2} \leq C|u|_{2}^{\frac{4-p}{2}}|v|_{2}^{\frac{p}{2}}\left|\partial_{x} u\right|_{2}^{p} \quad \text { for } 0 \leq p \leq 4,
$$

with a constant $C$. It follows that

$$
\left|v_{j}\right|_{2}^{2}+\left|\partial_{x} u_{j}\right|_{2}^{2} \leq C\left|u_{j}\right|_{2}^{\frac{4-p}{2}}\left(\left|v_{j}\right|_{2}^{2}+\left|\partial_{x} u_{j}\right|_{2}^{2}\right)^{\frac{3 p}{4}} \leq C\left(\left|v_{j}\right|_{2}^{2}+\left|\partial_{x} u_{j}\right|_{2}^{2}\right)^{\frac{3 p}{4}}
$$

or

$$
\left(\left|v_{j}\right|_{2}^{2}+\left|\partial_{x} u_{j}\right|_{2}^{2}\right)\left(1-C\left(\left|v_{j}\right|_{2}^{2}+\left|\partial_{x} u_{j}\right|_{2}^{2}\right)^{\frac{3 p-4}{4}}\right) \leq 0
$$

This implies that

$$
1 \leq C\left(\left|v_{j}\right|_{2}^{2}+\left|\partial_{x} u_{j}\right|_{2}^{2}\right)^{\frac{3 p-4}{4}}
$$

But it contradicts $\left|v_{j}\right|_{2}^{2}+\left|\partial_{x} u_{j}\right|_{2}^{2} \rightarrow 0$, because $p>\frac{4}{3}$. Consequently, $\inf _{j}\left|u_{j}\right|_{p+2}^{p+2}>0$.

Step 2. $u_{0} \neq 0$ a.e. 
Proof of Step 2. Let $\inf _{j}\left|u_{j}\right|_{p+2}^{p+2}=\alpha>0$. We estimate

$$
\begin{aligned}
\alpha & \leq\left|u_{j}\right|_{p+2}^{p+2}=\int_{\left|u_{j}\right| \geq \frac{1}{\epsilon}}\left|u_{j}\right|^{p+2}+\int_{\left|u_{j}\right| \leq \epsilon}\left|u_{j}\right|^{p+2}+\int_{\epsilon<\left|u_{j}\right|<\frac{1}{\epsilon}}\left|u_{j}\right|^{p+2} \\
& \leq \int_{\left|u_{j}\right| \geq \frac{1}{\epsilon}} \frac{\left|u_{j}\right|^{p+2+\gamma}}{\left|u_{j}\right|^{\gamma}}+\epsilon^{p} \int_{\left|u_{j}\right| \leq \epsilon}\left|u_{j}\right|^{2}+\left(\frac{1}{\epsilon}\right)^{p+2} \mu\left(\left|u_{j}\right|>\epsilon\right) \\
& \leq \epsilon^{\gamma} \int_{\left|u_{j}\right| \geq \frac{1}{\epsilon}}\left|u_{j}\right|^{p+2+\gamma}+\epsilon^{p} \int_{\left|u_{j}\right| \leq \epsilon}\left|u_{j}\right|^{2}+C_{\epsilon} \mu\left(\left|u_{j}\right|>\epsilon\right)
\end{aligned}
$$

where $0<\gamma<4-p$. Since $p+\gamma<4$, we have

$$
\int_{\left|u_{j}\right| \geq \frac{1}{\epsilon}}\left|u_{j}\right|^{2+p+\gamma} \leq \int_{\mathbb{R}^{2}}\left|u_{j}\right|^{2+p+\gamma} \leq C\left(\left|u_{j}\right|_{2}^{2}+\left|v_{j}\right|_{2}^{2}+\left|\partial_{x} u_{j}\right|_{2}^{2}\right)^{\frac{2+p+\gamma}{2}} \leq C_{1}
$$

and

$$
\int_{\left|u_{j}\right| \geq \frac{1}{\epsilon}}\left|u_{j}\right|^{2} \leq \int_{\mathbb{R}^{2}}\left|u_{j}\right|^{2} \leq C_{2}
$$

where $C, C_{1}$ and $C_{2}$ are some constants. Choosing $\epsilon$ sufficiently small, we obtain

$$
\mu\left(\left|u_{j}\right|>\epsilon\right) \geq \frac{\alpha-\epsilon^{\gamma} C_{1}-\epsilon^{p} C_{2}}{C_{\epsilon}}=\delta>0 .
$$

It follows from Lemma 2.9 that $\mu\left(B \cap\left(\left|u_{0}\right|>\frac{\epsilon}{2}\right)\right)>\delta_{0}$ for the unit ball $B$ because $u_{j} \rightarrow u_{0}$ a.e. in $\mathbb{R}^{2}$. This implies that $u_{0} \neq 0$ a.e. in $\mathbb{R}^{2}$.

Step 3. $m=L_{c}^{1}\left(u_{0}\right)$.

Proof of Step 3. By Lemma 2.7, we deduce that

$$
Q\left(u_{j}\right)-Q\left(u_{j}-u_{0}\right)-Q\left(u_{0}\right) \longrightarrow 0,
$$

and

$$
L_{c}^{1}\left(u_{j}\right)-L_{c}^{1}\left(u_{j}-u_{0}\right)-L_{c}^{1}\left(u_{0}\right) \longrightarrow 0 .
$$

Now suppose that $Q\left(u_{0}\right)>0$. Then from the fact that $Q\left(u_{j}\right) \leq 0$ we obtain that $Q\left(u_{j}-u_{0}\right) \leq 0$ as $j \rightarrow \infty$. By the definition of $m$, it follows that $L_{c}^{1}\left(u_{j}-u_{0}\right) \geq m$. Since $L_{c}^{1}\left(u_{j}\right) \rightarrow m$, it follows from $(2.13)$ that $L_{c}^{1}\left(u_{0}\right) \leq 0$. That is,

$$
\frac{c}{2} \int_{\mathbb{R}^{2}} u_{0}^{2}+\frac{3 p-4}{6 p} \int_{\mathbb{R}^{2}}\left(v_{0}^{2}+\left(\partial_{x} u_{0}\right)^{2}\right) \leq 0 .
$$

This contradicts $u_{0} \neq 0$, a.e. Consequently, $Q\left(u_{0}\right) \leq 0$. Therefore,

$$
m \leq L_{c}^{1}\left(u_{0}\right) \leq \lim _{j \rightarrow \infty} \inf L_{c}^{1}\left(u_{j}\right)=m
$$

and $m=L_{c}^{1}\left(u_{0}\right)$.

Step 4. $Q\left(u_{0}\right)=0$.

Proof of Step 4. Suppose that $Q\left(u_{0}\right)<0$. We try to get a contradiction. Toward this end, we choose a small $\lambda>0$, and find that

$$
Q\left(\lambda u_{0}\right)=\lambda^{2} \int_{\mathbb{R}^{2}}\left(v_{0}^{2}+\left(\partial_{x} u_{0}\right)^{2}\right)-\frac{3 p}{2(p+1)(p+2)} \lambda^{p+2} \int_{\mathbb{R}^{2}} u_{0}^{p+2}>0 .
$$


It follows from the continuity that there exists $\lambda_{0} \in(0,1)$ such that $Q\left(\lambda_{0} u_{0}\right)=0$. Therefore, by the definition of $m$, it yields a contradiction in the following:

$$
\begin{aligned}
m & \leq L_{c}^{1}\left(\lambda_{0} u_{0}\right)=\lambda_{0}^{2}\left(\frac{c}{2} \int_{\mathbb{R}^{2}} u_{0}^{2}+\frac{3 p-4}{6 p} \int_{\mathbb{R}^{2}}\left(v_{0}^{2}+\left(\partial_{x} u_{0}\right)^{2}\right)\right. \\
& <\frac{c}{2} \int_{\mathbb{R}^{2}} u_{0}^{2}+\frac{3 p-4}{6 p} \int_{\mathbb{R}^{2}}\left(v_{0}^{2}+\left(\partial_{x} u_{0}\right)^{2}=L_{c}^{1}\left(u_{0}\right)=m .\right.
\end{aligned}
$$

Hence it proves that $Q\left(u_{0}\right)=0$.

Step 5. $u_{0}=\varphi_{c} \in G_{c}$.

Proof of Step 5. In fact, it follows from Step 3 and Step 4 that

$$
m=\sigma=\inf \left\{L_{c}(u) \mid u \neq 0, Q(u)=0\right\}=L_{c}\left(u_{0}\right) .
$$

Hence, there exists a constant $\lambda \in \mathbb{R}$ such that

$$
L_{c}^{\prime}\left(u_{0}\right)+\lambda Q^{\prime}\left(u_{0}\right)=0 .
$$

It remains to show that $\lambda=0$ so that $L_{c}^{\prime}\left(u_{0}\right)=0$. By using $Q\left(u_{0}\right)=0$, it yields that

$$
\begin{aligned}
0 & =\left\langle L^{\prime}\left(u_{0}\right)+\lambda Q^{\prime}\left(u_{0}\right), u_{0}\right\rangle \\
& =\int_{\mathbb{R}^{2}}\left(c u_{0}^{2}+\left(\partial_{x} u_{0}\right)^{2}+v_{0}^{2}\right)-\frac{1}{p+1} \int_{\mathbb{R}^{2}} u_{0}^{p+2} \\
& +\lambda\left(2 \int_{\mathbb{R}^{2}}\left(\left(\partial_{x} u_{0}\right)^{2}+v_{0}^{2}\right)-\frac{3 p}{2(p+1)} \int_{\mathbb{R}^{2}} u_{0}^{p+2}\right) \\
& =\int_{\mathbb{R}^{2}}\left(c u_{0}^{2}+(2 \lambda+1)\left(v_{0}^{2}+\left(\partial_{x} u_{0}\right)^{2}\right)\right)-\frac{2(p+2)}{3 p} \int_{\mathbb{R}^{2}}\left(v_{0}^{2}+\left(\partial_{x} u_{0}\right)^{2}\right) \\
& -\lambda(p+2) \int_{\mathbb{R}^{2}}\left(v_{0}^{2}+\left(\partial_{x} u_{0}\right)^{2}\right) \\
& =\int_{\mathbb{R}^{2}} c u_{0}^{2}+\left(\frac{p-4}{3 p}-\lambda p\right) \int_{\mathbb{R}^{2}}\left(v_{0}^{2}+\left(\partial_{x} u_{0}\right)^{2}\right) .
\end{aligned}
$$

It follows from $c>0$ that $\frac{p-4}{3 p}-\lambda p<0$. On the other hand, let $w^{\eta}(x, y)=$ $\eta^{\frac{3}{2}} u_{0}\left(\eta x, \eta^{2} y\right)$. It is easy to see that

$$
\begin{aligned}
0 & =\left\langle\left(L_{c}^{\prime}+\lambda Q^{\prime}\right)\left(u_{0}\right),\left.\frac{\partial w^{\eta}}{\partial \eta}\right|_{\eta=1}\right\rangle=\left.\partial_{\eta}\left(L_{c}+\lambda Q\right)\left(w^{\eta}\right)\right|_{\eta=1} \\
& =Q\left(u_{0}\right)+\lambda\left(2 \int_{\mathbb{R}^{2}}\left(v_{0}^{2}+\left(\partial_{x} u_{0}\right)^{2}\right)-\frac{9 p^{2}}{4(p+1)(p+2)} \int_{\mathbb{R}^{2}} u_{0}^{p+2}\right) .
\end{aligned}
$$

If $\lambda \neq 0$, then

$$
2 \int_{\mathbb{R}^{2}}\left(v_{0}^{2}+\left(\partial_{x} u_{0}\right)^{2}\right)-\frac{9 p^{2}}{4(p+1)(p+2)} \int_{\mathbb{R}^{2}} u_{0}^{p+2}=0 .
$$

Since $Q\left(u_{0}\right)=0$, it follows that

$$
0=2 \int_{\mathbb{R}^{2}}\left(v_{0}^{2}+\left(\partial_{x} u_{0}\right)^{2}\right)-\frac{3 p}{2} \int_{\mathbb{R}^{2}}\left(v_{0}^{2}+\left(\partial_{x} u_{0}\right)^{2}\right)=\frac{4-3 p}{2} \int_{\mathbb{R}^{2}} v_{0}^{2}+\left(\partial_{x} u_{0}\right)^{2}<0
$$


because $p>\frac{4}{3}$. Hence $\lambda=0$ and $L^{\prime}\left(u_{0}\right)=0$. On the other hand, suppose $u \in Y$ satisfies $L_{c}^{\prime}(u)=0$. We have

$$
Q(u)=\left.\partial_{\eta} L_{c}\left(u^{\eta}\right)\right|_{\eta=1}=\left\langle L_{c}^{\prime}(u),\left.\frac{d u^{\eta}}{d \eta}\right|_{\eta=1}\right\rangle=0
$$

where $u^{\eta}=\eta^{\frac{3}{2}} u\left(\eta x, \eta^{2} y\right)$. It follows from $(2.15)$ that $L_{c}\left(u_{0}\right) \leq L_{c}(u)$. This implies that $u_{0}=\varphi_{c} \in G_{c}$. The proof of Lemma 2.11 is complete.

To prove Theorem 1.1, we also need the following two lemmas.

Lemma 2.12. Let $1 \leq p<4$ and $\varphi_{c} \in G_{c}$. Then

$$
L_{c}\left(\varphi_{c}\right)=\inf \left\{L_{c}(u) \mid u \in Y, u \neq 0, \int_{\mathbb{R}^{2}} u_{x}^{2}=\int_{\mathbb{R}^{2}}\left(\partial_{x} \varphi_{c}\right)^{2}\right\}
$$

Proof. Since $K=L_{c}(u)-\frac{1}{2} \int_{\mathbb{R}^{2}} u_{x}^{2}$, it follows from Lemma 2.10 that

$$
\begin{aligned}
& \inf \left\{L_{c}(u) \mid u \in Y, u \neq 0, \int_{\mathbb{R}^{2}} u_{x}^{2}=\int_{\mathbb{R}^{2}}\left(\partial_{x} \varphi_{c}\right)^{2}\right\} \\
& =\inf \left\{K(u)+\frac{1}{2} \int_{\mathbb{R}^{2}}\left(\partial_{x} \varphi_{c}\right)^{2} \mid u \in Y, u \neq 0, \int_{\mathbb{R}^{2}} u_{x}^{2}=\int_{\mathbb{R}^{2}}\left(\partial_{x} \varphi_{c}\right)^{2}\right\} \\
& =\inf \left\{K(u) \mid u \in Y, u \neq 0, \int_{\mathbb{R}^{2}} u_{x}^{2}=\int_{\mathbb{R}^{2}}\left(\partial_{x} \varphi_{c}\right)^{2}\right\}+\frac{1}{2} \int_{\mathbb{R}^{2}}\left(\partial_{x} \varphi_{c}\right)^{2} \\
& =\frac{1}{2} \int_{\mathbb{R}^{2}}\left(\partial_{x} \varphi_{c}\right)^{2}=L_{c}\left(\varphi_{c}\right) .
\end{aligned}
$$

Lemma 2.13. Let $1 \leq p<4$ and $\varphi_{c} \in G_{c}$. Then

$$
L_{c}\left(\varphi_{c}\right)=\inf \left\{L_{c}(u) \mid u \in Y, u \neq 0, \int_{\mathbb{R}^{2}} u^{p+2}=\int_{\mathbb{R}^{2}} \varphi_{c}^{p+2}\right\} .
$$

Proof.

$$
\begin{aligned}
& L_{c}\left(\varphi_{c}\right)=\inf \left\{L_{c}(u) \mid u \in Y, u \neq 0, \int_{\mathbb{R}^{2}} u^{p+2}=\int_{\mathbb{R}^{2}} \varphi_{c}^{p+2}\right\} \\
& =\frac{1}{2} \inf \left\{\int_{\mathbb{R}^{2}}\left(c u^{2}+v^{2}+u_{x}^{2}\right) \mid u \in Y, u \neq 0, \int_{\mathbb{R}^{2}} u^{p+2}=\int_{\mathbb{R}^{2}} \varphi_{c}^{p+2}\right\} \\
& -\frac{1}{(p+1)(p+2)} \int_{\mathbb{R}^{2}} \varphi_{c}^{p+2}=\frac{1}{2} I_{c}\left(\varphi_{c}\right)-\frac{1}{(p+1)(p+2)} \int_{\mathbb{R}^{2}} \varphi_{c}^{p+2}=L_{c}\left(\varphi_{c}\right) .
\end{aligned}
$$

\section{INVARIANT SETS}

In this section we construct some invariants for the flow of the KP equation (1.1). Using those invariant properties of the solution and a virial identity which has been showed in $\mathrm{TuFa}$, we are able to show the blow-up result in Section 4 . Toward this end, we begin to define the invariants in the following:

$$
\begin{aligned}
& K_{1}^{c}=\left\{u \in Y \mid u \neq 0, L_{c}(u)<L_{c}\left(\varphi_{c}\right), Q(u) \geq 0\right\}, \\
& K_{2}^{c}=\left\{u \in Y \mid u \neq 0, L_{c}(u)<L_{c}\left(\varphi_{c}\right), Q(u)<0\right\},
\end{aligned}
$$




$$
\begin{aligned}
& R_{1}^{c}=\left\{u \in Y \mid u \neq 0, L_{c}(u)<L_{c}\left(\varphi_{c}\right), \int_{\mathbb{R}^{2}} u_{x}^{2} \leq \int_{\mathbb{R}^{2}}\left(\partial_{x} \varphi_{c}\right)^{2}\right\}, \\
& R_{2}^{c}=\left\{u \in Y \mid u \neq 0, L_{c}(u)<L_{c}\left(\varphi_{c}\right), \int_{\mathbb{R}^{2}} u_{x}^{2}>\int_{\mathbb{R}^{2}}\left(\partial_{x} \varphi_{c}\right)^{2}\right\}, \\
& J_{1}^{c}=\left\{u \in Y \mid u \neq 0, L_{c}(u)<L_{c}\left(\varphi_{c}\right), \int_{\mathbb{R}^{2}} u^{p+2} \geq \int_{\mathbb{R}^{2}} \varphi_{c}^{p+2}\right\}, \\
& J_{2}^{c}=\left\{u \in Y \mid u \neq 0, L_{c}(u)<L_{c}\left(\varphi_{c}\right), \int_{\mathbb{R}^{2}} u^{p+2}<\int_{\mathbb{R}^{2}} \varphi_{c}^{p+2}\right\} .
\end{aligned}
$$

The following lemma is crucial to obtain the blow-up result.

Lemma 3.1 (Invariant sets). Suppose $1 \leq p<4$ and $c>0$. Let $u_{0}$ be the initial data such that the corresponding solution $u(t)$ of $K P$ equation $(1.1)$ is in $C([0, T) ; Y)$ for some $T>0$ and satisfies $E(u(t))=E\left(u_{0}\right)$ and $V(u(t))=V\left(u_{0}\right)$ for $0 \leq t<T$. Then

a) $\frac{4}{3}<p<4, u_{0} \in K_{i}^{c}$ implies that $u(t) \in K_{i}^{c}, \forall 0 \leq t<T$,

b) $u_{0} \in R_{i}^{c}$ implies that $u(t) \in R_{i}^{c}, \forall 0 \leq t<T$, and

c) $u_{0} \in J_{i}^{c}$ implies that $u(t) \in J_{i}^{c}, \forall 0 \leq t<T$ where $i=1,2$. Moreover, if $u_{0} \in K_{2}^{c}$, then $Q(u(t))<-\frac{3 p}{2}\left(L_{c}\left(\varphi_{c}\right)-L_{c}\left(u_{0}\right)\right)$ for $0 \leq t<T$.

Proof. Here we only consider the invariance of $K_{2}^{c}$, since for $K_{1}^{c}$ the proof is similar and the proof of invariance of $R_{1}^{c}$ and $J_{i}^{c}$ are also similar due to Lemmas 2.12 and 2.13 .

Let $u_{0} \in K_{2}^{c}$. Since $E(u(t))=E\left(u_{0}\right)$ and $V(u(t))=V\left(u_{0}\right)$, we have

$$
L_{c}(u(t))=E(u(t))+c V(u(t))=L_{c}\left(u_{0}\right)<L_{c}\left(\varphi_{c}\right) .
$$

Suppose $u\left(t_{0}\right) \notin K_{2}^{c}$ for some $t_{0} \in(0, T)$; that is, $Q\left(u\left(t_{0}\right)\right) \geq 0$. By $Q(u(0))=$ $Q\left(u_{0}\right)<0$ and the continuity of $Q(u(t))$ with respect to $t$, there exists $t_{1} \in\left(0, t_{0}\right]$ such that $Q\left(u\left(t_{1}\right)\right)=0$. Therefore, applying Lemma 2.11 yields a contradiction

$$
L_{c}\left(\varphi_{c}\right)>L_{c}\left(u\left(t_{1}\right)\right) \geq \inf \left\{L_{c}(u) \mid u \neq 0, Q(u)=0\right\}=L_{c}\left(\varphi_{c}\right) .
$$

This implies that $u(t) \in K_{2}^{c}$ for $0 \leq t<T$. To prove the final inequality, we use the definition of $m$ and the fact

$$
m=\inf \left\{L_{c}(u) \mid u \in Y, u \neq 0, Q(u)=0\right\}=L_{c}\left(\varphi_{c}\right)
$$

which is proved in Lemma 2.11.

Suppose $u_{0} \in K_{2}^{c}$. Then we have $u(t) \in K_{2}^{c}$, i.e. $L_{c}(u(t))<L_{c}\left(\varphi_{c}\right)$ and $Q(u(t))<$ 0 for $t \geq 0$. Since

$$
Q(\lambda u)=\lambda^{2} \int_{\mathbb{R}^{2}}\left(v^{2}+u_{x}^{2}\right)-\frac{3 p}{2(p+1)(p+2)} \lambda^{p+2} \int_{\mathbb{R}^{2}} u^{p+2}>0
$$

for some sufficiently small $\lambda>0$, there exists $\lambda_{0} \in(0,1)$, such that $Q\left(\lambda_{0} u\right)=0$ and

$$
\begin{aligned}
L_{c}\left(\varphi_{c}\right) & \leq L_{c}\left(\lambda_{0} u\right)=\lambda_{0}^{2}\left(\frac{c}{2} \int_{\mathbb{R}^{2}} u^{2}+\frac{3 p-4}{6 p} \int_{\mathbb{R}^{2}}\left(v^{2}+u_{x}^{2}\right)\right) \\
& <\frac{c}{2} \int_{\mathbb{R}^{2}} u^{2}+\frac{3 p-4}{6 p} \int_{\mathbb{R}^{2}}\left(v^{2}+u_{x}^{2}\right)=L_{c}^{1}(u(t)) .
\end{aligned}
$$

Therefore, $Q(u(t))<-\frac{3 p}{2}\left(L_{c}\left(\varphi_{c}\right)-L_{c}\left(u_{0}\right)\right)$. 
Remark. If $u_{0} \in K_{2}^{c} \cap J_{2}^{c}$, then it follows from the conservation laws $E(u)$ and $V(u)$ that $\|u(t)\|_{Y} \leq C\left(\varphi_{c},\left\|u_{0}\right\|_{Y}\right)$. Consequently, the solution $u(t)$ of the KP equation is bounded globally for $t \geq 0$; blow-up cannot occur in finite time in $Y\left(\mathbb{R}^{2}\right)$. However, we do have a blow-up solution which is only due to the transverse dispersion; we will state it in the next section.

\section{Finite BLOW-UP time}

In Section 1, we mention that the solution of (1.1) blows up in finite time if the initial data $u_{0}$ satisfies $E\left(u_{0}\right)<0$ and $p \geq 4$ TuFa, Sa1]. Using the invariant sets, we are able to extend this blow-up result to allow the energy $E$ to be even positive and also $\frac{4}{3} \leq p<4$. We start to prove the case of $p>\frac{4}{3}$ and consider the critical case $p=\frac{4}{3}$ later.

Theorem 4.1 (Improved blow-up). Let $\frac{4}{3}<p<4$ with $p=\frac{n_{1}}{n_{2}}$, where $n_{1}$ is an even integer and $n_{2}$ an odd integer. Assume

i) Let $u_{0} \in Y$ and $y u_{0} \in L^{2}$, and

ii) $u_{0} \in K_{2}^{c} \cap R_{2}^{c} \cap J_{2}^{c}$.

Let $u$ be the solution of the KP equation (1.1) in $C([0, T) ; Y)$ with $u(0)=u_{0}$, conserved energy $E$ and momentum $V$. Then there exists a blow-up time $T_{0}<\infty$ such that

$$
\lim _{t \rightarrow T_{0}^{-}}\left|\partial_{y} u(t)\right|_{2}=\infty .
$$

Proof. If $u(t)$ remains in $Y$, we define $I(t)=\int_{\mathbb{R}^{2}} y^{2} u^{2}(x, y, t) d x d y$, where $u$ is the solution of the KP equation (1.1). Using the virial identity in TuFa, WaAbSe, Sa1] yields

$$
\begin{aligned}
\frac{d^{2} I(t)}{d t^{2}} & =4 p E(u(t))+2(4-p) \int_{\mathbb{R}^{2}} v^{2}-2 p \int_{\mathbb{R}^{2}} u_{x}^{2} \\
& =8\left(Q(u(t))-\int_{\mathbb{R}^{2}} u_{x}^{2}+\frac{p}{(p+1)(p+2)} \int_{\mathbb{R}^{2}} u^{p+2}\right) .
\end{aligned}
$$

It follows from Lemma 3.1 that

$$
\begin{aligned}
\frac{d^{2} I(t)}{d t^{2}}<8\left(-\frac{3 p}{2}\left(L_{c}\left(\varphi_{c}\right)\right.\right. & \left.-L_{c}\left(u_{0}\right)\right)-\int_{\mathbb{R}^{2}}\left(\partial_{x} \varphi_{c}\right)^{2} \\
& \left.+\frac{p}{(p+1)(p+2)} \int_{\mathbb{R}^{2}} \varphi_{c}^{p+2}\right) \\
& =-12 p \epsilon_{0}
\end{aligned}
$$

where $\epsilon_{0}=L_{c}\left(\varphi_{c}\right)-L_{c}\left(u_{0}\right)$. In the last step of the above proof, we used the fact

$$
\int_{\mathbb{R}^{2}}\left(\partial_{x} \varphi_{c}\right)^{2}=\frac{p}{(p+1)(p+2)} \int_{\mathbb{R}^{2}} \varphi_{c}^{p+2}
$$

which can be obtained from $\left.\frac{\partial}{\partial \lambda} L_{c}\left(\varphi_{c}^{\lambda}\right)\right|_{\lambda=1}=0$ where $\varphi_{c}^{\lambda}=\lambda \varphi_{c}(\lambda x, \lambda y)$. Therefore, it follows that $\lim _{t \rightarrow T_{0}} I(t)=0$ for some $T_{0}<\infty$, And the blow-up result can be deduced from the conserved momentum $V(u)$ and the classical inequality

$$
|u|_{2}^{2} \leq 2|y u|_{2}\left|\partial_{y} u\right|_{2}
$$

The proof of Theorem 4.1 is complete. 
Remark 1. If $u_{0} \in R_{2}^{c} \cap J_{2}^{c}$ and $p \geq 2$, then $E\left(u_{0}\right)>0$. This can be verified as follows.

Since

$$
\int_{\mathbb{R}^{2}}\left(\partial_{x} u_{0}\right)^{2}>\int_{\mathbb{R}^{2}}\left(\partial_{x} \varphi_{c}\right)^{2}, \int_{\mathbb{R}^{2}} u_{0}^{p+2}<\int_{\mathbb{R}^{2}} \varphi_{c}^{p+2}
$$

and

$$
\int_{\mathbb{R}^{2}}\left(\partial_{x} \varphi_{c}\right)^{2}=\frac{p}{(p+1)(p+2)} \int_{\mathbb{R}^{2}} \varphi_{c}^{p+2}
$$

we have

$$
\begin{aligned}
E\left(u_{0}\right) & =\frac{1}{2} \int_{\mathbb{R}^{2}}\left(\partial_{x} u_{0}\right)^{2}+\frac{1}{2} \int_{\mathbb{R}^{2}} v_{0}^{2}-\frac{1}{(p+1)(p+2)} \int_{\mathbb{R}^{2}} u_{0}^{p+2} \\
& >\frac{1}{2} \int_{\mathbb{R}^{2}}\left(\partial_{x} \varphi_{c}\right)^{2}+\frac{1}{2} \int_{\mathbb{R}^{2}} v_{0}^{2}-\frac{1}{(p+1)(p+2)} \int_{\mathbb{R}^{2}} \varphi_{c}^{p+2} \\
& =\int_{\mathbb{R}^{2}} v_{0}^{2}+\frac{p-2}{2 p} \int_{\mathbb{R}^{2}}\left(\partial_{x} \varphi_{c}\right)^{2} \geq \frac{1}{2} \int_{\mathbb{R}^{2}} v_{0}^{2}>0
\end{aligned}
$$

Remark 2. Since $E\left(\varphi_{c}\right)=\frac{3 p-4}{6 p}\left(\left|\partial_{x} \varphi_{c}\right|_{2}^{2}+\left|\partial_{x}^{-1} \partial_{y} \varphi_{c}\right|_{2}^{2}\right)$ and $E\left(\varphi_{c}\right)>0$ when $p>\frac{4}{3}$, we are able to choose $u_{0}=\varphi_{c}^{\lambda}$ which is sufficiently close to $\varphi_{c}$ such that $E\left(u_{0}\right)>0$.

Remark 3. A similar blow-up result can be obtained for a generalized KadomtsevPetiashvili (KP) equation in the three-dimensional case [Liu]

$(\mathrm{KP}-3 \mathrm{D})$

$$
\left\{\begin{array}{l}
u_{t}+u_{x x x}+u^{p} u_{x}=v_{y}+w_{z} \\
v_{x}=u_{y} \\
w_{x}=u_{z}
\end{array} \quad(x, y) \in \mathbb{R}^{3}, t \geq 0 .\right.
$$

The solution of KP-3D blows up in finite time for all $p \geq 1$.

Next, we consider the critical case $p=\frac{4}{3}$.

Theorem 4.2. Let $p=\frac{4}{3}$. Assume

i) $u_{0} \in Y, \partial_{y} u_{0} \in L^{2}$ and $y u_{0} \in L^{2}$,

ii) $u_{0} \in R_{2}^{c} \cap J_{2}^{c}$, and

iii) $E\left(u_{0}\right)<0$.

Let $u$ be the solution of the KP equation (1.1) in $C([0, T) ; Y)$ with $u(0)=u_{0}$ and satisfy $E(u(t))=E\left(u_{0}\right)$ and $V(u(t))=V\left(u_{0}\right)$. Then there exists a blow-up time $T_{0}<\infty$ such that

$$
\lim _{t \rightarrow T_{0}}\left|\partial_{y} u(t)\right|_{2}=\infty
$$

In order to show Theorem 4.2, we need the following basic lemma.

Lemma 4.3. Let $p=\frac{4}{3}$. Then $E\left(\varphi_{c}\right)=0$ for $\varphi_{c} \in G_{c}$, and

$$
2 \int_{\mathbb{R}^{2}}\left(\partial_{x} \varphi_{c}\right)^{2}=\frac{4-p}{(p+1)(p+2)} \int_{\mathbb{R}^{2}} \varphi_{c}^{p+2} .
$$

Proof. Define $\phi_{c}^{\lambda}=\lambda \varphi_{c}(\lambda x, \lambda y)$. It can be easily verified that

$$
0=\left.\frac{d}{d \lambda} L_{c}\left(\varphi_{c}^{\lambda}\right)\right|_{\lambda=1}=\int_{\mathbb{R}^{2}}\left(\partial_{x} \varphi_{c}\right)^{2}-\frac{p}{(p+1)(p+2)} \int_{\mathbb{R}^{2}} \varphi_{c}^{p+2} ;
$$


that is,

$$
2 \int_{\mathbb{R}^{2}}\left(\partial_{x} \varphi_{c}\right)^{2}=\frac{2 p}{(p+1)(p+2)} \int_{\mathbb{R}^{2}} \varphi_{c}^{p+2}=\frac{4-p}{(p+1)(p+2)} \int_{\mathbb{R}^{2}} \varphi_{c}^{p+2}
$$

because $p=\frac{4}{3}$. To prove $E\left(\varphi_{c}\right)=0$, it follows from the fact $Q\left(\varphi_{c}\right)=0$ that

$$
\begin{aligned}
& E\left(\varphi_{c}\right)+c V\left(\varphi_{c}\right) \\
& =\frac{c}{2} \int_{\mathbb{R}^{2}}+\frac{1}{2} \int_{\mathbb{R}^{2}}\left(\left(\partial_{x}^{-1} \partial_{y} \varphi_{c}\right)^{2}+\left(\partial_{x} \varphi_{c}\right)^{2}\right)-\frac{1}{(p+1)(p+2)} \int_{\mathbb{R}^{2}} \varphi_{c}^{p+2} \\
& =\frac{c}{2} \int_{\mathbb{R}^{2}}+\frac{3 p-4}{6 p} \int_{\mathbb{R}^{2}}\left(\left(\partial_{x}^{-1} \partial_{y} \varphi_{c}\right)^{2}+\left(\partial_{x} \varphi_{c}\right)^{2}\right)=\frac{c}{2} \int_{\mathbb{R}^{2}}=c V\left(\varphi_{c}\right) .
\end{aligned}
$$

This implies that $E\left(\varphi_{c}\right)=0$.

Proof of Theorem 4.2. By Lemma 3.1, the solution $u(t) \in R_{2}^{c}$ and $u(t) \in J_{2}^{c}$ for $0 \leq t<T$; that is, $|u(t)|_{p+2}^{p+2}<\left|\varphi_{c}\right|_{p+2}^{p+2}$ and $\left|\partial_{x} u(t)\right|_{2}^{2}>\left|\partial_{x} \varphi_{c}\right|_{2}^{2}$ where $\varphi_{c} \in G_{c}$. It follows from the viral identity and Lemma 4.3 that

$$
\begin{aligned}
I^{\prime \prime}(t) & =16 E(u(t))-8 \int_{\mathbb{R}^{2}} u_{x}^{2}+\frac{4(4-p)}{(p+1)(p+2)} \int_{\mathbb{R}^{2}} u^{p+2} \\
& <16 E\left(u_{0}\right)-8\left|\partial_{x} \varphi_{c}\right|_{2}^{2}+\frac{4(4-p)}{(p+1)(p+2)} \int_{\mathbb{R}^{2}} \varphi_{c}^{p+2}=16 E\left(u_{0}\right) .
\end{aligned}
$$

Since $E\left(u_{0}\right)<0$, the inequality (4.4) implies $\lim _{t \rightarrow T_{0}} I(t)=0$ for a finite time $T_{0}<\infty$. Therefore,

$$
\lim _{t \rightarrow T_{0}}\left|\partial_{y} u(t)\right|_{2}=\infty
$$

This completes the proof of Theorem 4.2.

\section{Strong instability of Solitary Waves}

In the proof of Theorem 4.1, we find that one assumption for the blow-up $u_{0} \in$ $R_{2}^{c} \cap J_{2}^{c}$ can be relaxed to $u_{0} \in N_{c}$, for $2<p<4$, where

$$
N_{c}=\left\{u \in Y \mid u \neq 0, L_{c}(u)<L_{c}\left(\varphi_{c}\right), P(u)>0\right\}
$$

and

$$
P(u)=\int_{\mathbb{R}^{2}} u_{x}^{2}-\frac{p}{(p+1)(p+2)} \int_{\mathbb{R}^{2}} u^{p+2} .
$$

A strong instability of solitary-wave solutions $\varphi_{c}$. can be obtained in the following.

Theorem 5.1. Let $2<p<4$ with $p=\frac{n_{1}}{n_{2}}$, where $n_{1}$ is an even integer and $n_{2}$ an odd integer. Suppose $\varphi_{c} \in Y$ is the solitary-wave solution of the KP equation (1.1) with $c>0$. For any $\delta>0$, there is an initial data $u_{0} \in Y$ with $\left\|u_{0}-\varphi_{c}\right\|_{Y}<\delta$, such that the solution $u$ of (1.1) with $u(0)=u_{0}$ blows up in finite time. More precisely,

$$
\lim _{t \rightarrow T^{-}}\left|\partial_{y} u(t)\right|_{2}=\infty .
$$

To prove Theorem 5.1, we need the following lemmas.

Lemma 5.2. Let $\tau=\inf \left\{L_{c}(u) \mid u \neq 0, P(u)=0\right\}$. Suppose $2<p<4$. Then $\varphi_{c} \in G_{c}$ if and only if $P\left(\varphi_{c}\right)=0$ and $\tau=L_{c}\left(\varphi_{c}\right)$.

Since the proof is similar to that of Lemma 2.11, we only give a sketch of the proof. 
Sketch of the proof. $P\left(\varphi_{c}\right)=0$ is given by the equation (4.4). Let

$$
L_{c}^{2}(u)=L_{c}(u)-\frac{1}{p} P(u)
$$

Then we have

$$
L_{c}^{2}(u)=\frac{c}{2} \int_{\mathbb{R}^{2}} u^{2}+\frac{1}{2} \int_{\mathbb{R}^{2}} v^{2}+\frac{p-2}{2 p} \int_{\mathbb{R}^{2}} u_{x}^{2}>0
$$

when $2<p$. It can be shown that $\tau=m_{1}$ where

$$
m_{1}=\inf \left\{L_{c}^{2}(u) \mid u \in Y, u \neq 0, P(u) \leq 0\right\} .
$$

In fact, suppose $P(u)<0$. There exists a small number $\lambda \in(0,1)$ such that $P\left(\lambda_{0} u\right)=0$ and

$$
\tau \leq L_{c}\left(\lambda_{0} u\right)=\lambda_{0}^{2}\left(\frac{c}{2} \int_{\mathbb{R}^{2}} u^{2}+\frac{1}{2} \int_{\mathbb{R}^{2}} v^{2}+\frac{p-2}{2 p} \int_{\mathbb{R}^{2}} u_{x}^{2}\right)<L_{c}^{2}(u) .
$$

This implies that $\tau=m_{1}$. On the other hand, since $L_{c}^{2}(u)>0$, there exists a minimizing sequence of $\left\{u_{j}\right\}, u_{j} \neq 0, \forall j$ such that $P\left(u_{j}\right) \leq 0, L_{c}^{2}\left(u_{j}\right) \rightarrow m_{1}$, and $u_{j} \rightarrow u_{0}$ weakly in $V\left(\mathbb{R}^{2}\right)$ as well as in $L^{2+p}$. In next step, we need to show $\inf _{j}\left|u_{j}\right|_{p+2}^{p+2}>0$, so that $u_{0} \neq 0$, a.e. In fact, if $\left|u_{j}\right|_{p+2}^{p+2} \rightarrow 0$, then by $P\left(u_{j}\right) \leq 0$, we deduce that $\left|\partial_{x} u_{j}\right|_{2} \rightarrow 0$. It follows from the anisotropic Sobolev embedding that

$$
\left|\partial_{x} u_{j}\right|_{2}^{2} \leq\left|u_{j}\right|_{2}^{\frac{4-p}{2}}\left|v_{j}\right|_{2}^{\frac{p}{2}}\left|\partial_{x} u_{j}\right|_{2}^{p} \leq C\left|\partial_{x} u_{j}\right|_{2}^{p}
$$

Because $p>2$, it yields a contradiction $1 \leq C\left|\partial_{x} u_{j}\right|_{2}^{p-2}$. To show the rest of the proof, we use the weak convergence lemma (Lemma 2.7) and follow the proofs in Step 3, Step 4 and Step 5 of Lemma 2.11.

By the proof of Lemma 3.1, we are also able to obtain the following lemma.

Lemma 5.3. $N_{c}$ is invariant for the solution of the KP equation (1.1). That is, $u_{0} \in N_{c}$ implies that the corresponding solution $u(t) \in N_{c}$ for $t \geq 0$.

Now by using Lemmas 5.2 and 5.3 , it is easy to obtain the following lemma which is crucial to prove the instability of solitary waves (Theorem 5.1).

Lemma 5.4. Let $2<p<4$. Assume

i) $u_{0} \in Y$ and $y u_{0} \in L^{2}$, and

ii) $u_{0} \in K_{2}^{c} \cap N_{c}$.

Let $u$ be the solution of the KP equation (1.1) in $C([0, T) ; Y)$ with $u(0)=u_{0}, E(u(t))$ $=E\left(u_{0}\right)$ and $V(u(t))=V\left(u_{0}\right)$. Then there exists a blow-up time $T_{0}<\infty$ such that

$$
\lim _{t \rightarrow T_{0}}\left|\partial_{y} u(t)\right|_{2}=\infty .
$$

Proof. It follows from the proof of Theorem 4.1 that

$$
\frac{d^{2} I}{d t^{2}}=8(Q(u(t))-P(u(t))) .
$$

By Lemma 5.3, we obtain that $P(u(t))>0$, for $t \geq 0$. Hence

$$
\frac{d^{2} I}{d t^{2}}<8 Q(u(t))<-12 p \epsilon_{0}
$$

and a blow-up time $T_{0}$ can easily be deduced. Using this lemma we are able to prove Theorem 5.1. 
Proof of Theorem 5.1. We define $u_{0}(x, y)=\chi_{\omega}(x, y)=\sigma^{a}(\omega) \varphi_{\omega}\left(\sigma^{b} x, \sigma^{e} y\right)$, where $\sigma^{2 a-b-e}(\omega)=\frac{\left|\varphi_{c}\right|_{2}^{2}}{\left|\varphi_{\omega}\right|_{2}^{2}}, b=\frac{a p}{2}+\eta, e=a p-\epsilon-\eta$ with $\epsilon, \eta>0$ and $a<0$. It is easy to see that $\left|\chi_{\omega}\right|_{2}^{2}=\left|\varphi_{c}\right|_{2}^{2}, \chi_{c}=\varphi_{c}$, and $\sigma(c)=1$. On the other hand,

$$
\left.\sigma^{\prime}(\omega)\right|_{\omega=c}=\frac{-d^{\prime \prime}(c)}{d^{\prime}(c)(2 a-b-e)}=\frac{-d^{\prime \prime}(c)}{\frac{1}{2}\left|\varphi_{c}\right|_{2}^{2}\left(\frac{4-3 p}{2} a-\epsilon\right)}>0
$$

for small $\epsilon$. By the smooth of $\chi_{\omega}$ with respect to $\omega$, it is found that $\chi_{\omega}$ is sufficiently close to $\varphi_{c}$ as $\omega \rightarrow c$. Let $u(t)$ be the solution of the KP equation (1.1) with $u(0)=$ $u_{0}$. By the smooth of $\varphi_{\omega}$ [BoSa2], the solution $u \in C([0, T) ; Y)$ has the conserved energy $E$ and the momentum $V$. By Lemma 5.3, it remains to show $\chi_{\omega} \in K_{2}^{c} \cap N_{c}$ as $\omega \rightarrow c$. More precisely, $Q\left(\chi_{\omega}\right)<0, P\left(\chi_{\omega}\right)>0$, and $L_{c}\left(\chi_{\omega}\right)<L_{c}\left(\varphi_{c}\right)$ for some $\omega>c$. By a simple computation, we have

$$
Q\left(\chi_{\omega}\right)=\left(\frac{1}{2} \sigma^{2 a+e-3 b}(\omega)+\sigma^{2 a+b-e}(\omega)-\frac{3}{2} \sigma^{a(p+2)-e-b}(\omega)\right)\left|\varphi_{\omega}\right|_{2}^{2} .
$$

At $\omega=c$, we have $\left.Q\left(\chi_{\omega}\right)\right|_{\omega=c}=0$ and the differentiation of $Q\left(\chi_{\omega}\right)$ with respect to $\omega$ at $\omega=c$ yields

$$
\begin{aligned}
\left.\frac{d Q\left(\chi_{\omega}\right)}{d \omega}\right|_{\omega=c} & =\left(\frac{1}{2}(2 a+e-3 b)+(2 a+b-e)-\frac{3}{2}(a(p+2)-e-b)\right) \sigma^{\prime}(c)\left|\partial_{x} \varphi_{c}\right|_{2}^{2} \\
& =\left(e+b-\frac{3}{2} a p\right) \sigma^{\prime}(c)\left|\partial_{x} \varphi_{c}\right|_{2}^{2}=-\epsilon \sigma^{\prime}(c)\left|\partial_{x} \varphi_{c}\right|_{2}^{2}<0 .
\end{aligned}
$$

This implies that $Q\left(\chi_{\omega}\right)<Q\left(\chi_{c}\right)=Q\left(\varphi_{c}\right)=0$ for $\omega>c$. Next, we have

$$
P\left(\chi_{\omega}\right)=\sigma^{1+2 b}\left(1-\sigma^{a p-2 b}\right)\left|\partial_{x} \varphi_{\omega}\right|_{2}^{2}
$$

and at $\omega=c$

$$
\left.\frac{d P}{d \omega}\right|_{\omega=c}=(2 b-a p) \sigma^{\prime}(c)\left|\partial_{x} \varphi_{c}\right|_{2}^{2}=2 \eta \sigma^{\prime}(c)\left|\partial_{x} \varphi_{c}\right|_{2}^{2}>0 .
$$

This implies that $P\left(\chi_{\omega}\right)>P\left(\chi_{c}\right)=P\left(\varphi_{c}\right)=0$ for $\omega>c$.

Finally, we need to show $L_{c}\left(\chi_{\omega}\right)<L_{c}\left(\varphi_{c}\right)$ for $\omega>c$. Let $L_{\omega}(u)=E(u)+\omega V(u)$. A simple computation yields

$$
\begin{aligned}
L_{\omega} & =\frac{\omega}{2} \sigma^{2 a-b-e}\left|\varphi_{\omega}\right|_{2}^{2}+\frac{1}{2} \sigma^{2 a-3 b+e}\left|\partial_{x}^{-1} \partial_{y} \varphi_{\omega}\right|_{2}^{2} \\
& +\frac{1}{2} \sigma^{2 a+b-e}\left|\partial_{x} \varphi_{\omega}\right|_{2}^{2}-\frac{1}{(p+1)(p+2)} \sigma^{a(p+2)-b-e}\left|\varphi_{\omega}\right|_{p+2}^{p+2} \\
& =\left(\frac{4-p}{4 p} \sigma^{2 a-b-e}+\frac{1}{4} \sigma^{2 a-3 b+e}+\frac{1}{2} \sigma^{2 a+b-e}-\frac{1}{p} \sigma^{a(p+2)-b-e}\right)\left|\partial_{x} \varphi_{\omega}\right|_{2}^{2} .
\end{aligned}
$$

In the above computation, we use the fact that

$$
\begin{array}{cl}
\left|\partial_{x}^{-1} \partial_{y} \varphi_{\omega}\right|_{2}^{2}=\frac{1}{2}\left|\partial_{x} \varphi_{\omega}\right|_{2}^{2}, \quad \frac{\omega}{2}\left|\varphi_{\omega}\right|_{2}^{2}=\frac{p-4}{4 p}\left|\partial_{x} \varphi_{\omega}\right|_{2}^{2}, \\
\frac{1}{(p+1)(p+2)}\left|\varphi_{\omega}\right|_{p+2}^{p+2}=\frac{1}{p}\left|\partial_{x} \varphi_{\omega}\right|_{2}^{2}, \quad \text { and } \quad d(\omega)=\frac{1}{2}\left|\partial_{x} \varphi_{\omega}\right|_{2}^{2} .
\end{array}
$$

Differentiation of $L_{\omega}\left(\chi_{\omega}\right)$ with respect to $\omega$ at $\omega=c$ yields the formulae

$$
\left.\frac{d L_{\omega}\left(\chi_{\omega}\right)}{d \omega}\right|_{\omega=c}=d^{\prime}(c)=\frac{1}{2}\left|\varphi_{c}\right|_{2}^{2}
$$


and

$$
\begin{aligned}
& \left.\frac{d^{2} L_{\omega}\left(\chi_{\omega}\right)}{d \omega^{2}}\right|_{\omega=c}=d^{\prime \prime}(c)+\left(\frac{1}{2}(2 a+b-e)^{2}+\frac{4-p}{4 p}(2 a-b-e)^{2}\right. \\
& \left.+\frac{1}{4}(2 a-3 b+e)^{2}-\frac{1}{p}(a(p+2)-b-e)^{2}\right) 2 d(c)\left(\sigma^{\prime}(c)\right)^{2} \\
& =d^{\prime \prime}(c)+\left(\frac{1}{2}(2 a+b-e)^{2}+\frac{4-p}{4 p}(2 a-b-e)^{2}\right. \\
& \left.+\frac{1}{4}(2 a-3 b+e)^{2}-\frac{1}{p}(a(p+2)-b-e)^{2}\right) \frac{2}{(2 a-b-e)^{2}} \frac{4-3 p}{4-p} d^{\prime \prime}(c) \\
& =\frac{2(4-3 p)}{(2 a-b-e)^{2}(4-p)} g(\epsilon, \eta) d^{\prime \prime}(c),
\end{aligned}
$$

where

$$
\begin{aligned}
g(\epsilon, \eta) & =\frac{1}{2}\left(\frac{4-p}{2} a+\epsilon+2 \eta\right)^{2}-\frac{(4-p)^{2}}{4 p(3 p-4)}\left(\frac{4-3 p}{2} a \epsilon\right)^{2} \\
& +\frac{1}{4}\left(\frac{4-3 p}{2} a-4 \epsilon-\eta\right)^{2}-\frac{1}{p}\left(\frac{4-p}{2} a+\epsilon\right)^{2} .
\end{aligned}
$$

Now, we claim $\left.\frac{d^{2} L_{\omega}\left(\chi_{\omega}\right)}{d \omega^{2}}\right|_{\omega=c}<0$. Since $d^{\prime \prime}(c)<0$ and $4-3 p<0$, it suffices to show that $g(\epsilon, \eta)<0$ for small $\epsilon$ and $\eta$. In fact, $g(0,0)=0$,

$$
\frac{\partial g}{\partial \eta}(0, \eta)=2\left(\frac{4-p}{2} a+2 \eta\right)-\frac{1}{2}\left(\frac{4-3 p}{2} a-\eta\right)
$$

and $\frac{\partial g}{\partial \eta}(0,0)=\frac{1}{4}(12-p) a<0$. This implies that $g(0, \eta)<g(0,0)=0$ for small $\eta>0$ and therefore $g(\epsilon, \eta)<0$ for small $\epsilon>0$ and $\eta>0$. It follows from $\left.\frac{d^{2} L_{\omega}\left(\chi_{\omega}\right)}{d \omega^{2}}\right|_{\omega=c}<0$ that

$$
\begin{aligned}
L_{c}\left(\chi_{\omega}\right) & =L_{\omega}\left(\chi_{\omega}\right)+V\left(\omega_{\omega}\right)(c-\omega)=L_{\omega}\left(\chi_{\omega}\right)+V\left(\varphi_{c}\right)(c-\omega) \\
& <L_{c}\left(\varphi_{c}\right)+\left.\frac{d}{d \omega} L_{\omega}\left(\chi_{\omega}\right)\right|_{\omega=c}(\omega-c)+V\left(\varphi_{c}\right)(c-\omega) \\
& =L_{c}\left(\varphi_{c}\right)+V\left(\varphi_{c}\right)(\omega-c)+V\left(\varphi_{c}\right)(c-\omega)=L_{c}\left(\varphi_{c}\right)
\end{aligned}
$$

for $\omega$ sufficiently near $c$. Therefore the result of strong instability of solitary waves can be obtained by Lemma 5.4.

\section{REFERENCES}

[AlPeSa] Alexander, J.C., Pego, R. L., and Sachs, R. L., On the transverse instability of solitary eaves in the Kadomtsev-Petviashvili equation, Phys. Lett. A 226 (1997), 187-192. MR 97j:35132

[AbSe] Ablowitz, M., and Segur, H., Solitons and the Inverse Scattering Transform, SIAM, Philadelphia, 1981. MR 84a:35251

[BeBoMa] Benjamin, T., Bona, J., and Mahony, J., Model equations for long waves in nonlinear dispersive systems, Phil. Trans. Roy. Soc. London A272 (1972), 47-78. MR 55:898

[BeIlNi] Besov, O. V., Il'in, V. P., and Nikolskiı̌, S. M., Integral Representations of Functions and Imbedding Theorems, Vol. I, J. Wiley, New York, 1978. MR 80f:46030a

[Bo] Bona, J., On the stability of solitary waves, Proc. Roy. Soc. London A344 (1975), 363-374. MR 52:7292 
[BoDoKa] Bona, J., Dougalis, V. A. and Karakashian, O. A., Fully discrete Galerkin methods for the Korteweg-de Vries equation, Comput. Math. Appl. Ser. A 12 (1986), 859-884. MR 87j:65108

[Bou] Bourgain, J., On the Cauchy problem for the Kadomtsev-Petviashvili equations, Geom. Funct. Anal. 3(4) (1993), 315-341. MR 94d:35142

[BoLiu] Bona, J. and Liu, Yue, Instability of solitary waves of the Kadomtsev-Petviashvili equation in three dimensions, preprint.

[BoSoSt] Bona, J., Souganidis, P. and Strauss, W., Stability and instability of solitary waves of Korteweg-de Vries type, Proc. Roy. Soc. London Ser. A 411 (1987), 395-412. MR 88m:35128

[BoSa1] de Bouard A. and Saut, J.C., Solitary waves of generalized Kadomtsev-Petviashvili equations, Ann. Inst. H. Poincaré Anal. Non Linéaire 14 (1997), 211-236. MR 98a:35115

[BoSa2] de Bouard A. and Saut, J. C., Symmetries and decay of the generalized KadomtsevPetviashvili solitary waves, SIAM J. Math. Anal. 28 (1997), 1064-1085. MR 99c:35208

[BoSa3] de Bouard A. and Saut, J. C., Remarks on the stability of generalized KP solitary waves, Contemp. Math., 200, Amer. Math. Soc., Providence, R.I., 1996, 75-84. MR 97a:35157

[BrLi1] Brezis, H., and Lieb, E., A relation between pointwise convergence of functions and convergence of functionals, Proc. Amer. Math. Soc. 88 (1983), 486-490. MR 84e:28003

[BrLi2] Brezis, H., and Lieb, E., Minimum action solutions of some vector field equations, Comm. Math. Phys. 96 (1984), 97-113. MR 86d:35045

[FoSu] Fokas, A. S. and Sung, L. Y., On the solvability of the $N$-wave, DaveyStewartson and Kadomtsev-Petviashvili equations, Inverse Problems 8 (1992), 673708. MR 93h:35177

[IsMeSt] Isaza, P., Mejía, J., and Stallbohm, V., Local solution for the Kadomtsev-Petviashvili equation in $R^{2}$, J. Math. Anal. Appl. 196 (1995), 566-587. MR 96j:35217

[KaPe] Kadomtsev, B. B. and Petviashvili, V. I., On the stability of solitary waves in weakly dispersive media, Soviet Phys. Dokl. 15(6) (1970), 539-541.

$[\mathrm{KePoVe}]$ Kenig, C. E., Ponce, G. and Vega, L., Well-posedness and scattering results for the generalized Korteweg-de Vries equation via the contraction principle, Comm. Pure Appl. Math. 46 (1993), 527-620. MR 94h:35229

[LiuWa] Liu, Yue and Wang, Xiao-Ping, Nonlinear stability of solitary waves of a generalized Kadomtsev-Petviashvili equation, Comm. Math. Phys. 183 (1997), 253-266. MR 99b:35184

[Liu] Liu, Yue, Blow up and instability of solitary waves to a generalized KadomtsevPetviashvili equation in three dimensions, in preparation.

[PeYa] Petviashvili, V. and Yan'kov, V., Solitons and turbulence, in B. B. Kadomtsev (ed.) Rev. Plasma Phys. XIV, (1989), 1-62.

[Sa1] Saut, J., Remarks on the generalized Kadomtsev-Petviashvili equations, Indiana Math. J. 42(3) (1993), 1011-1026. MR 95j:35200

[Sa2] Saut, J., Recent results on the generalized Kadomtsev-Petviashvili equations, Acta Appl. Math. 39 (1995), 477-487. MR 96a:35178

[SoSt] Souganidis, P. and Strauss, W., Instability of nonlinear of a class of dispersive solitary waves, Proc. Roy. Soc. Edinburgh, 114A, (1990) 195-212. MR 92a:35143

[To] Tom, M. M., On the Generalized Kadomtsev-Petviashvili equation, Contemp. Math., Amer. Math. Soc., 1996, 193-210. MR 97e:35165

[TuFa] Turitsyn, S. and Falkovitch, G., Stability of magneto elastic solitons and self-focusing of sound in antiferromagnet, Soviet Phys. JETP 62 (1985), 146-152.

[Uk] Ukai, S., Local solutions of the Kadomtsev-Petviashvili equation, J. Fac. Sci. Univ. Tokyo, Sect. IA Math. 36 (1989), 193-209. MR 90i:35270

[WaAbSe] Wang, X-P., Ablowitz, M., and Segur, H., Wave collapse and instability of solitary waves of a generalized Kadomtsev-Petviashvili equation, Physica D 78 (1994), 241265. MR 95g:35182

[We] Weinstein, M., On the solitary traveling wave of the generalized Korteweg-de Vries equation, Amer. Math. Soc., Lectures in Appl. Math. 23 (1986). MR 88a:35218

[Wi] Wickerhauser, M. V., Inverse scattering for the heat operators and evolutions in $2+1$ variables, Comm. Math. Phys. 108 (1987), 67-89. MR 87m:35224 
[Za] Zakharov, V., On the stochastization of one dimensional chains of nonlinear oscilla-

tors, Sov. Phys. JETP 38 (1974), 108-110.

[Zh] Zhou, X., Inverse scattering transform for the time dependent Schödinger equation with applications to the KPI equation, Comm. Math. Phys. 128 (1990), 551-564. MR 91j:34138

Department of Mathematics, The University of Texas at Arlington, Arlington, TEXAS 76019

E-mail address: liu@math.uta.edu 